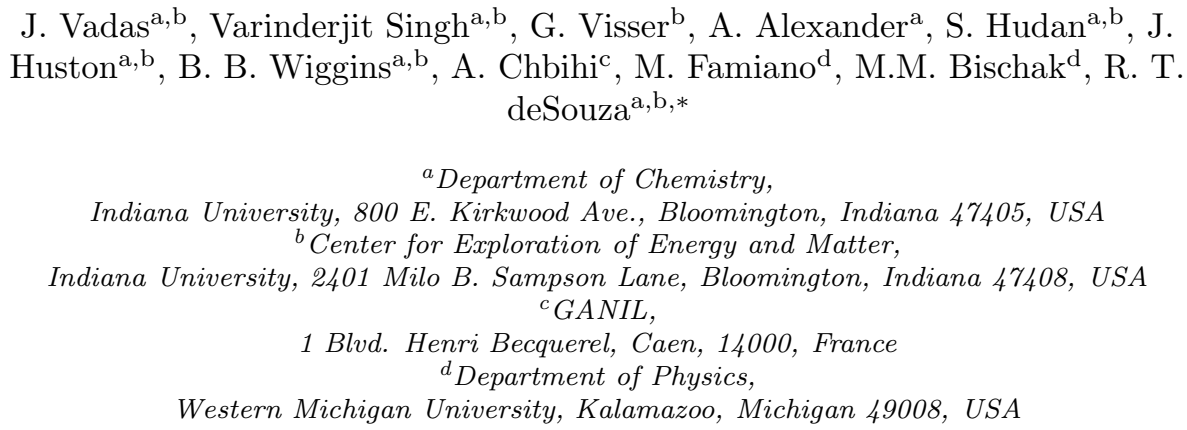

\title{
High-rate axial-field ionization chamber for particle identification of radioactive beams
}

The design, construction and performance characteristics of a simple axial-field ionization chamber suitable for identifying ions in a radioactive beam are presented. Optimized for use with low-energy radioactive beams $(<5 \mathrm{MeV} / \mathrm{A})$ the detector presents only three $0.5 \mu \mathrm{m} / \mathrm{cm}^{2}$ foils to the beam in addition to the detector gas. A fast charge sensitive amplifier (CSA) integrated into the detector design is also described. Coupling this fast CSA to the axial field ionization chamber produces an output pulse with a risetime of 60-70 ns and a fall time of 100 ns, making the detector capable of sustaining a relatively high rate and providing a time resolution of 6-8 ns. Tests with an $\alpha$ source establish the detector energy resolution as $\sim 8 \%$ for an energy deposit of $\sim 3.5 \mathrm{MeV}$. The energy resolution with beams of 2.5 and $4.0 \mathrm{MeV} / \mathrm{A}^{39} \mathrm{~K}$ ions and the dependence of the energy resolution on beam intensity is measured. At an instantaneous rate of 3 $\mathrm{x} 10^{5}$ ions/s the energy resolution has degraded to $14 \%$ with a pileup of $12 \%$. The good energy resolution of this detector at rates up to $3 \times 10^{5}$ ions $/ \mathrm{s}$ makes it an effective tool in the characterization of low-energy radioactive beams.

Keywords: ionization chamber, particle identification, radioactive beam

*desouza@indiana.edu 


\section{Introduction}

The development of radioactive isotope beams (RIBs) has enabled the investigation of nuclei away from $\beta$-stability [1-4], which is crucial in understanding nucleosynthesis in exotic astrophysical environments [3], as well as the structure of exotic nuclei $[3,4]$. RIBs can be produced by a variety of techniques including projectile fragmentation [5], ISOL [6], and photofission [7]. As the primary nuclear reaction produces a distribution of product nuclei, to provide a useful secondary radioactive beam, it is necessary to select the nuclide of interest from this distribution. This separation is typically accomplished through electromagnetic means [8]. Often, however, this separation does not produce a pure beam, and other reaction products with similar mass-to-charge ratios contaminate the beam. An effective way to address this challenge is to identify each ion in the beam on a particle-by-particle basis. A commonly used approach to accomplish this is through measurement of the energy loss $(\Delta \mathrm{E})$ and time-of-flight (TOF) of each ion [9].

The $\Delta \mathrm{E}$ information is often obtained from gas ionization chambers [10] used in transmission mode. The uniform thickness achieveable with these detectors, as well as their robustness against radiation damage are key factors in their utility. A common geometry of an ion chamber utilizes an electric field transverse to the incident ion direction [11], with a Frisch grid used to remove the dependence of the pulse amplitude on position. In this geometry of ion chambers, however, the drift time of the electrons in the direction transverse to the beam direction typically limits the rate to $\sim 10^{4}$ ions/s. To overcome this limitation of a slow response time, an axial field design can be employed. Axial field ionization chambers have been successfully employed as $\Delta \mathrm{E}$ detectors for heavy-ions since the 1980's $[12,13]$. Recently, the high rate capability of this design has been exploited at both high [14] and low energies [15] by using multiple tilted electrodes in the beam path to identify reaction products in a radioactive beam. When the incident energy is sufficiently high $(\sim 100 \mathrm{MeV} / \mathrm{A})$ thin metallized foils have been utilized for the electrodes. In contrast, at low 


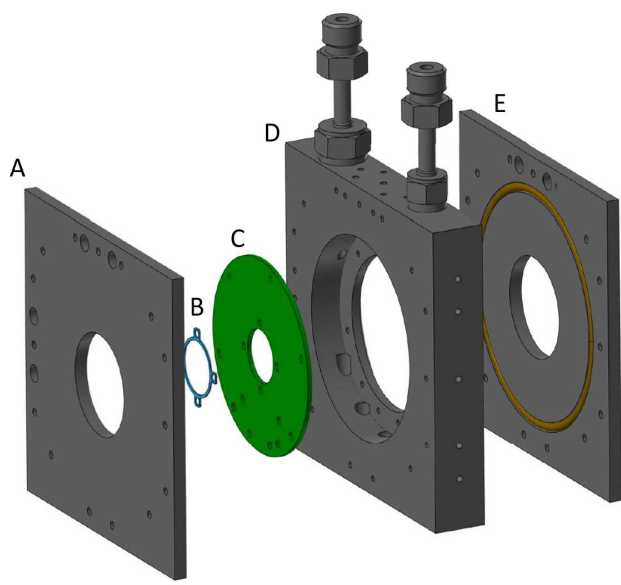

Figure 1: CAD drawing of the Rare Ion Purity Detector in an exploded view. A: window frame. B: anode ring. C: PCB. D: RIPD. body E: window frame. The mylar windows and central anode foil are omitted for clarity.

incident energies $(\sim 5 \mathrm{MeV} / \mathrm{A})$ the electrodes consist of wire harps. Both designs involve the insertion of multiple electrode planes (15-24) into the beam path, a source of considerable scattering and energy loss for the incident ion. In the subsequent sections, we describe the development and characterization of a simple high-rate axial-field ionization chamber, designated the Rare Ion Purity Detector (RIPD) which inserts a minimal amount of material into the beam path. The compact, minimalist design of this detector is capable of operating at a rate only slightly less $\left(3 \times 10^{5} \mathrm{pps}\right)$ than that of the more complex, tilted multi-electrode geometry ( $\left.7 \times 10^{5} \mathrm{pps}\right)[15]$ while presenting a minimal source of additional scattering of the incident beam.

\section{Physical Configuration}

The principal motivation in developing this detector was investigation of fusion for neutron-rich light ion beams at energies near the fusion barrier [16, 17]. Due to the low energy beams utilized in these experiments, particular attention was given to the total thickness of the detector in order to minimize the beam divergence and energy straggling incurred by inserting the detector into the 
beam path. Prior experience established that minimizing scattering of the beam was essential. This requirement meant eliminating any wire planes in the beam path. To implement the simplest axial field geometry while minimizing the electron collection time, a central anode is used with the metallized windows serving as cathodes.

A CAD drawing of RIPD is shown in Fig. 1. The detector is comprised of an aluminum body, two stainless steel window plates, and a thin central anode foil. The anode is coupled to a charge sensitive amplifier (CSA) housed inside the aluminum body. The body measures approximately $11 \mathrm{~cm} \mathrm{x} 11 \mathrm{~cm}$ transverse to the beam direction and $2.0 \mathrm{~cm}$ thick along the beam axis. The window plates are $5 \mathrm{~mm}$ thick and have a $38 \mathrm{~mm}$ diameter opening for the beam to pass through, over which $0.5 \mu \mathrm{m}$ aluminized mylar is epoxied. These mylar foils serve to contain the gas within the active volume and act as cathodes. The window plates are sealed to the body of the detector using O-rings. No support wires are used with these windows to minimize scattering of the incident beam. Using this geometry, it is possible to operate the detector at a pressure of 30 torr of $\mathrm{CF}_{4}$ for several days without any noticeable degradation in the window performance. Repeatedly filling the detector with gas also did not cause a noticeable deterioration in the mylar window. The $0.5 \mu \mathrm{m}$ mylar anode foil, which is doubly aluminized, is mounted on a $2.0 \mathrm{~cm}$ diameter stainless steel ring. This ring is attached to an annular printed circuit board (PCB) with an inner diameter of $2.0 \mathrm{~cm}$ and an outer diameter of $7.4 \mathrm{~cm}$. The position of the PCB is chosen so that the distance between the anode foil and each cathode foil is $1 \mathrm{~cm}$. Holes in the PCB allow gas to flow between the two halves of the detector. This arrangement of the anode and CSA in close proximity minimizes any additional capacitance at the input of the CSA.

\section{Charge Sensitive Amplifier}

As a key goal in the design of this axial field ionization chamber is its ability to maintain good energy resolution while sustaining a high rate with minimimum pileup of signals, it was necessary to develop a fast, low-noise charge 


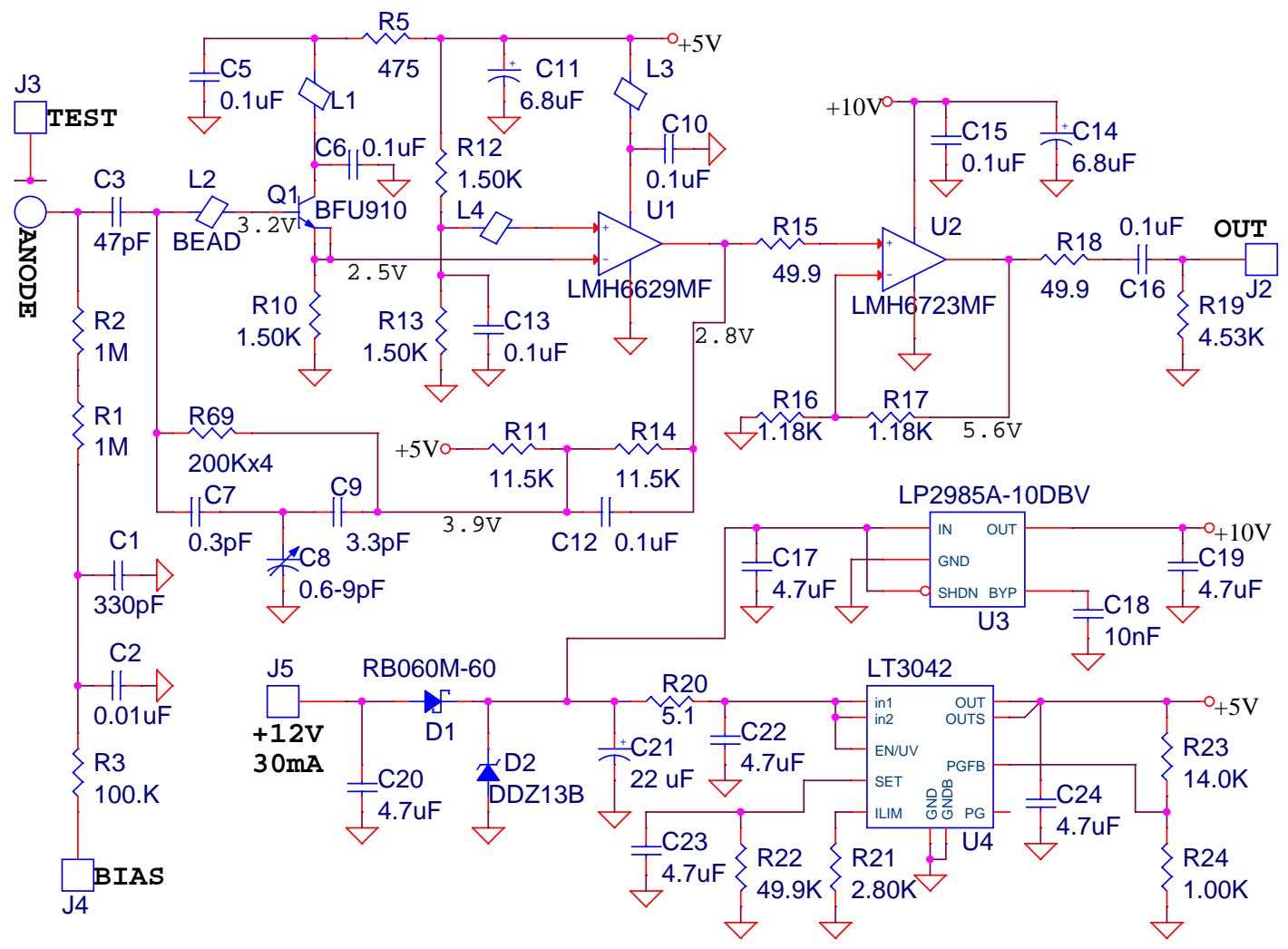

Figure 2: Schematic of the charge sensitive amplifier for RIPD. 
sensitive amplifier. Ionization of the detector gas induced by a beam particle traversing RIPD quasi-instantaneously produces an ionization track in the detector. Electrons in this track migrate under the influence of the applied electric field and are collected at the central anode. It should be noted that in contrast to the tilted foil design $[14,15]$ the electric field in RIPD does not move the electrons away from the path of the ionizing beam. Thus, in comparison to the tilted foil geometry the effects of recombination and screening are expected to be larger. This disadvantage is offset by the simplicity of the present design. Carbon tetrafluoride $\left(\mathrm{CF}_{4}\right)$ was chosen as the detector gas due to its high electron drift velocity [18]. Based upon the electron drift velocity for a reduced field of $1 \mathrm{kV} \mathrm{cm}^{-1} \mathrm{~atm}^{-1}$, a rise time of $100 \mathrm{~ns}$ is anticipated. This charge collection time defined one of the necessary characteristics of the CSA. To minimize the impact of stray capacitance, the CSA was situated on the PCB as close as practically possible to the central anode. The input capacitance of the detector was calculated to be $2.25 \mathrm{pF}$, which was confirmed by measurement.

The CSA is a new design that is intended to enable high count rates from low capacitance detectors. The design was initially intended to resolve beam contaminants arriving simultaneously with multiple beam ions in a $12 \mathrm{nS}$ interval. Achieving this separation required a bandwidth exceeding $80 \mathrm{MHz}$. With a feedback resistor of $18 \mathrm{k} \Omega$, a bandwidth of 90 to $100 \mathrm{MHz}$ was achieved. Changes in the experimental requirements led us to increase the gain of the CSA, consequently decreasing the bandwidth, by increasing the feedback resistor value. The schematic of the CSA is shown in Fig.2. The first opamp (U1) provides most of the gain with only a small contribution to the overall noise. However, its bias current, input current noise, and input capacitance are too high for direct connection to the anode, so a SiGe microwave transistor (Q1) is added to serve as an input buffer. This particular transistor offers very high current gain (about 2000) and extremely low input capacitance, and is also very quiet. Choice of a JFET for this purpose was precluded by the bandwidth originally intended. The signal response of this composite amplifier is defined by the feedback network of R69 and $\mathrm{C} 7,8$, and 9 . R69 is actually 4 chip resistors totaling 


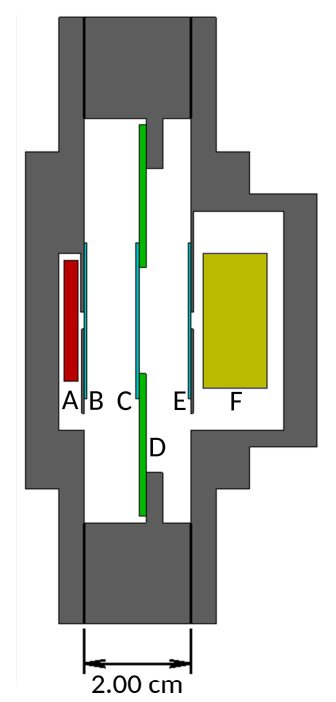

Figure 3: Cross-sectional view of the experimental setup to measure the energy loss of $\alpha$ particles. An ${ }^{241} \mathrm{Am} \alpha$ source was placed just upstream of the entrance foil in a cap that was made common with the active volume. Alpha particles that pass through the full length of the detector were then measured in a silicon surface barrier detector (SBD) placed just downstream of the exit window in a cap that was also made common with the active volume. A: ${ }^{241} \mathrm{Am} \alpha$ source. B: Entrance cathode foil. C: Anode foil. D: CSA. E: Exit cathode foil. F: SBD.

800 kohms, which were stacked end to end to minimize stray capacitance. The capacitors form a network with an equivalent capacitance adjustable from 0.1 to $0.3 \mathrm{pF}$. This network simplifies to just a RC single-pole response. The anode board also comprises an output buffer with a gain of 2 , two voltage regulators (U4 is remarkably low noise), level shift, and anode bias circuits. Minimizing the size ot the circuit was dictated by the need to minimize parasitics. This circuit was realized on a annular FR4 printed circuit board on which the RIPD anode was mounted. Excluding the bias and power circuit the CSA occupies an area of approximately $19 \mathrm{~mm}$ x $19 \mathrm{~mm}$. At $12 \mathrm{~V}$ the circuit consumes $24 \mathrm{ma}$.

\section{Experimental setup}

To characterize the performance of RIPD, the energy resolution for $\alpha$ particles from an ${ }^{241} \mathrm{Am}$ source was measured. To test RIPD with $\alpha$ particles, which have a low ionization density, it is necessary to operate RIPD at gas pressures 
that exceed the maximum pressure sustainable with the thin windows. For these tests, the entrance and exit window plates were replaced with flanges, as shown in Fig 3. The entrance flange allowed an ${ }^{241} \mathrm{Am}$ source to be situated prior to the entrance cathode foil but within the gas volume. Correspondingly, the exit flange allowed a silicon surface barrier detector (SBD) to be placed after the exit cathode foil within the gas volume. Both the ${ }^{241} \mathrm{Am}$ source and SBD had a collimation of $3 \mathrm{~mm}$. Triggering on the SBD signal associated with arrival of $\alpha$ particles selected particles that had traversed the entire thickness of RIPD. Using this configuration, signals in RIPD could be examined for pressures between 100 and 400 torr. At a pressure of 100 torr the $5.48 \mathrm{MeV} \alpha$ particles deposit just $680 \mathrm{keV}$ in the gas. This energy deposit increases to $\sim 3.5 \mathrm{MeV}$ at 400 torr.

\section{Signals}

A typical signal from the CSA at a pressure of 300 torr is shown in Fig. 4. The anode was biased to a potential of $+395 \mathrm{~V}$ to produce a reduced field of 1 $\mathrm{kV} \mathrm{cm}^{-1} \mathrm{~atm}^{-1}$. The signal time from baseline to peak is approximately $100 \mathrm{~ns}$ with a rise time of $60 \mathrm{~ns}$. Although the observed signal rise time corresponds to the convolution of the electron collection time and the CSA response, as the CSA response is fast $(<10 \mathrm{~ns})$, the observed signal principally reflects the electron collection time, consistent with the reported literature value for the electron drift velocity at the reduced field utilized. The CSA signal returns to baseline after $300 \mathrm{~ns}$. Thus the whole signal duration is under $500 \mathrm{~ns}$, which corresponds to a maximum calculated rate of $\sim 2 \times 10^{6}$ ions/second without pileup. The gain of the CSA is approximately $9.5 \mathrm{mV} / \mathrm{MeV}$. The noise of this signal is approximately $4 \mathrm{mV}$ peak-to-peak, corresponding to a signal-to-noise ratio of 5.7 .

To handle these fast signals, the development of a fast shaping amplifier was required. This requirement was realized by modifying an in-house octal shaper module which handles input signals of both polarities. With the fast shaper module shaping times between $100 \mathrm{~ns}$ and $800 \mathrm{~ns}$ in increments of $100 \mathrm{~ns}$ can be selected. The coarse gain is controlled by two 4-bit stages, while a fine 


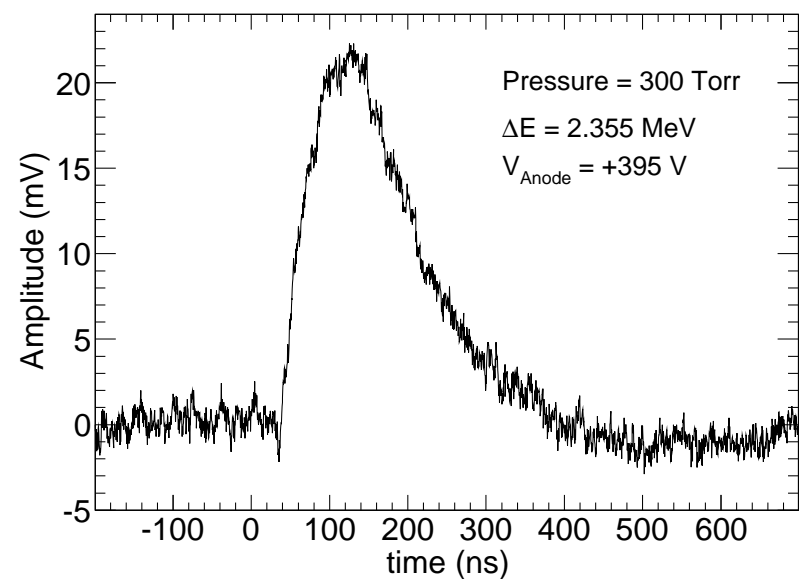

Figure 4: Typical signal from the CSA corresponding to the energy loss of an ${ }^{241} \mathrm{Am} \alpha$ particle with 300 torr of $\mathrm{CF}_{4}$ gas in the detector.

gain adjustment is provided using a 12-bit multiplying ADC. Another 12-bit multiplying ADC allows adjustment of the pole-zero. All of these parameters can be adjusted under computer control through a USB 2.0 interface. With a shaping time of $200 \mathrm{~ns}$ the fast shaping amplifier transforms the typical input CSA signal depicted in Fig.4 into a Gaussian-like pulse shown with an amplitude of $\sim 950 \mathrm{mV}$ and a peak-to-peak high frequency noise of $\sim 30 \mathrm{mV}$. Thus the shaping amplifier improves the signal-to-noise ratio to a value of approximately 31.

\section{Energy Resolution}

To determine the energy resolution of RIPD, the signal from the shaping amplifier was digitized by a CAEN V785 peak-sensing ADC. The energies of particles measured in RIPD was first calibrated using the measured energies in the SBD detector after accounting for the energy lost in the mylar foils [19]. The calibrated RIPD energy spectrum was used to determine the energy resolution by comparing the FWHM of the energy distribution to its centroid. The dependence of the energy resolution on the energy deposited in the gas volume is shown in Fig. 5. For energies up to $\sim 2 \mathrm{MeV}$, the absolute resolution is constant 


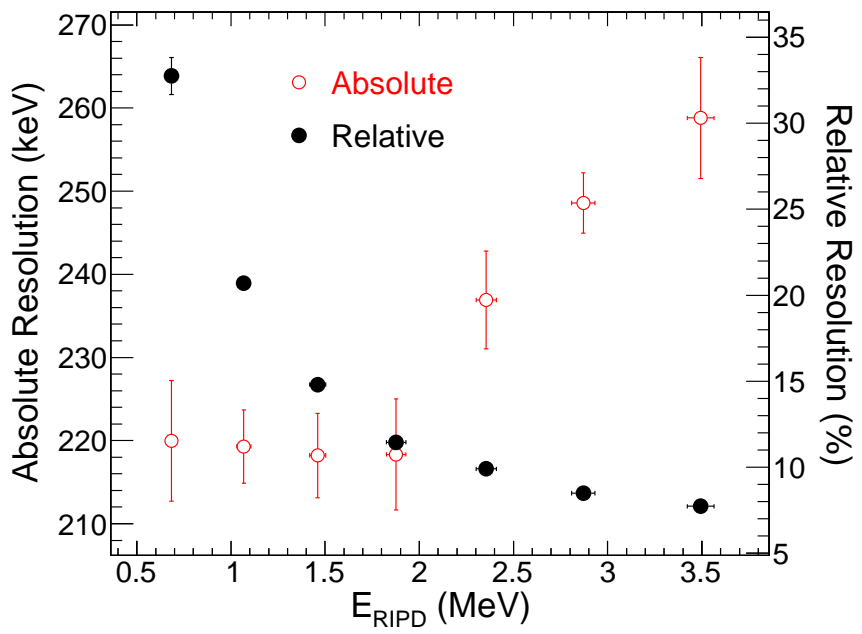

Figure 5: Resolution as a function of energy deposit in the gas volume of RIPD for $\alpha$ particles from a ${ }^{241}$ Am source.

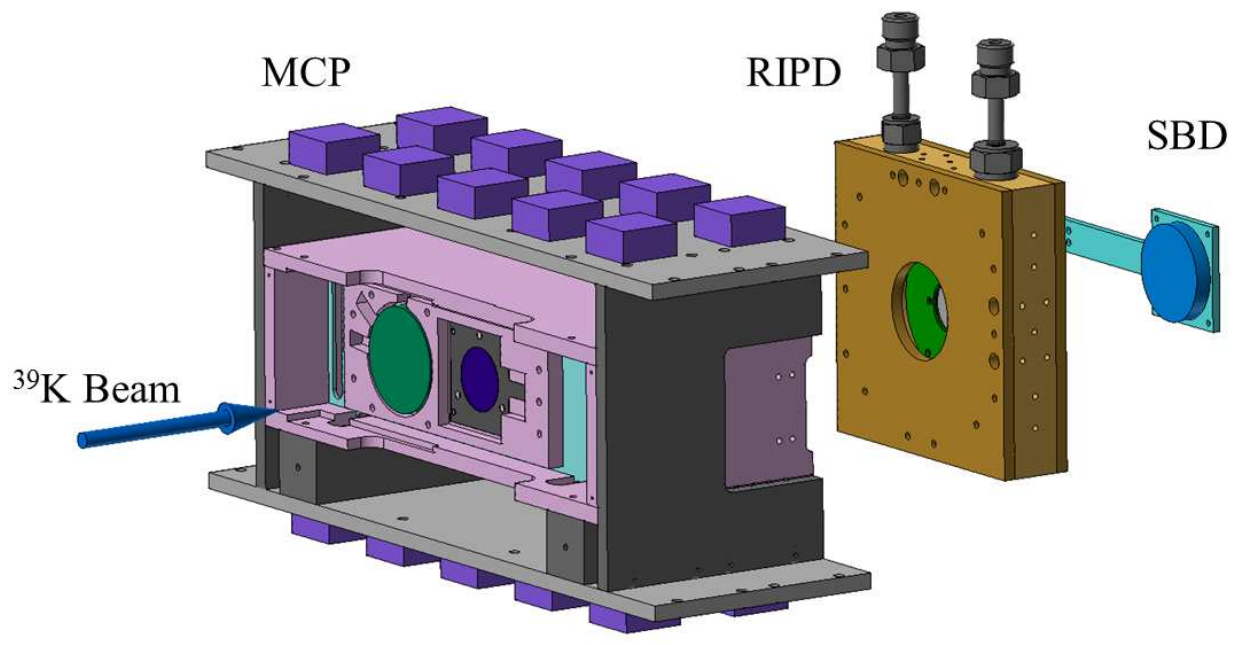

Figure 6: CAD representation of the experimental setup used to characterize RIPD with beam. 
at a value of approximately $220 \mathrm{keV}$, suggesting that for these energy deposits the electronic noise dominates the total noise. Above $2 \mathrm{MeV}$ the absolute resolution deteriorates reaching a value of $\sim 260 \mathrm{keV}$ at $3.5 \mathrm{MeV}$ of energy deposit. In the energy deposit interval measured the relative resolution decreases smoothly from $32 \%$ to $\sim 7.5 \%$. This $7.5 \%$ resolution can be understood as a combination of the signal-to-noise after the fast shaper as well as the variations in the path length of the measured $\alpha$ particles through the gas volume.

\section{Performance with Beam}

In order to characterize the performance of RIPD with beams of different intensity a test was conducted at the ReA3 facility at Michigan State University's National Superconducting Cyclotron Laboratory (NSCL). The ReA3 80 $\mathrm{MHz}$ linac, which can be used to accelerate either stable beams or radioactive ions produced by the NSCL's coupled cyclotron facility, was used to accelerate ${ }^{39} \mathrm{~K}$ to energies of $2.5 \mathrm{MeV} / \mathrm{A}$ and $4 \mathrm{MeV} / \mathrm{A}$. The beam was extracted from the charge breeding ion trap EBIT within 100 milliseconds at a repetition rate of $2 \mathrm{~Hz}$. This time structure results in the instantaneous rate experienced by any detector in the beam path being effectively a factor of five higher than the average rate. The experimental setup used is depicted in Fig. 6. The first element of the setup was a microchannel plate detector [20]. In this detector, passage of a beam particle through a $100 \mu \mathrm{g} / \mathrm{cm}^{2}$ carbon foil ejects electrons that are transported by crossed electric and magnetic fields to the surface of a chevron microchannel plate $(\mathrm{MCP})$. The fast response of the MCP results in each beam ion incident on the carbon foil being individually recorded. The rate at which the MCP triggers was recorded by a $250 \mathrm{MHz}$ VME scaler providing a measure of the beam rate. Approximately $44 \mathrm{~cm}$ downstream of the MCP, RIPD was mounted on a retractable arm. For low intensity beams a SBD situated immediately after RIPD was used to measure the residual energy of ions traversing RIPD. The SBD was retracted from the beam path when a high intensity beam was used. 


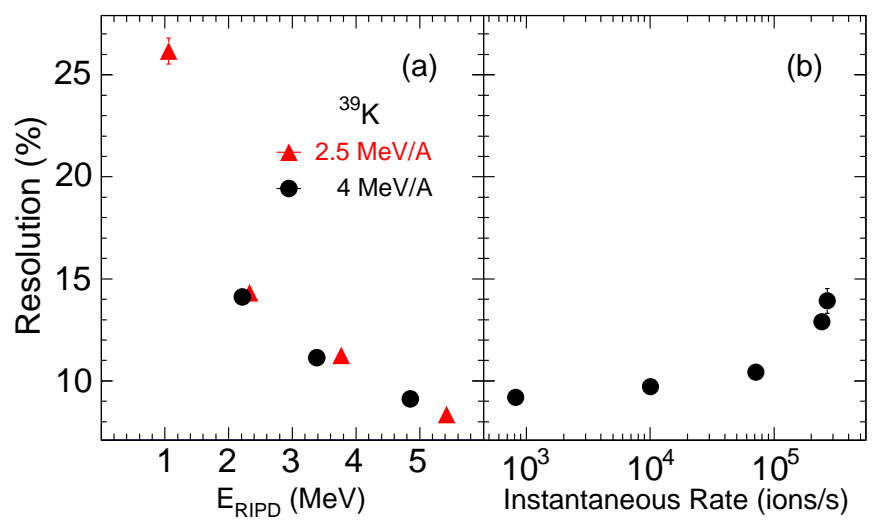

Figure 7: Panel a: Dependence of the energy resolution on the deposited energy in RIPD. Panel b: Impact on energy resolution of increased instantaneous beam rate.

Shown in Fig. 7a as the closed triangles is the energy resolution of the energy deposited in RIPD by $2.5 \mathrm{MeV} / \mathrm{A}{ }^{39} \mathrm{~K}$ ions traversing RIPD at nominal gas pressures of $5,10,15$, and 20 torr. The gas pressure and flow through the detector were maintained by a high quality gas handling system with a stability of 0.2 torr. With increasing gas pressure the energy deposit in the gas increases from approximately $1 \mathrm{MeV}$ to approximately $5 \mathrm{MeV}$. As the energy deposit in the gas increases the relative resolution of RIPD improves from $25 \%$ at the lowest energy deposit to approximately $9 \%$ at the highest energy deposit in reasonable agreement with the source testing results presented in Fig. 5. The electronic noise as well as the stability of the electronics were monitored during the measurement by injecting a calibration pulse into the charge sensitive amplifier at a low rate. The electronic noise was measured to be approximately $1 \%$, significantly less than the measured energy resolution of the detector. To determine the response of the energy resolution of RIPD to a change in the beam intensity, the beam intensity of a $4 \mathrm{MeV} / \mathrm{A}{ }^{39} \mathrm{~K}$ beam was increased from 800 ions $/ \mathrm{s}$ to $3 \times 10^{5}$ ions/s. The impact of the beam intensity on the energy resolution of RIPD is shown in Fig. 7b. With increasing beam intensity the measured energy resolution degrades from approximately $9 \%$ to $\sim 14 \%$ at the highest intensity measured. 


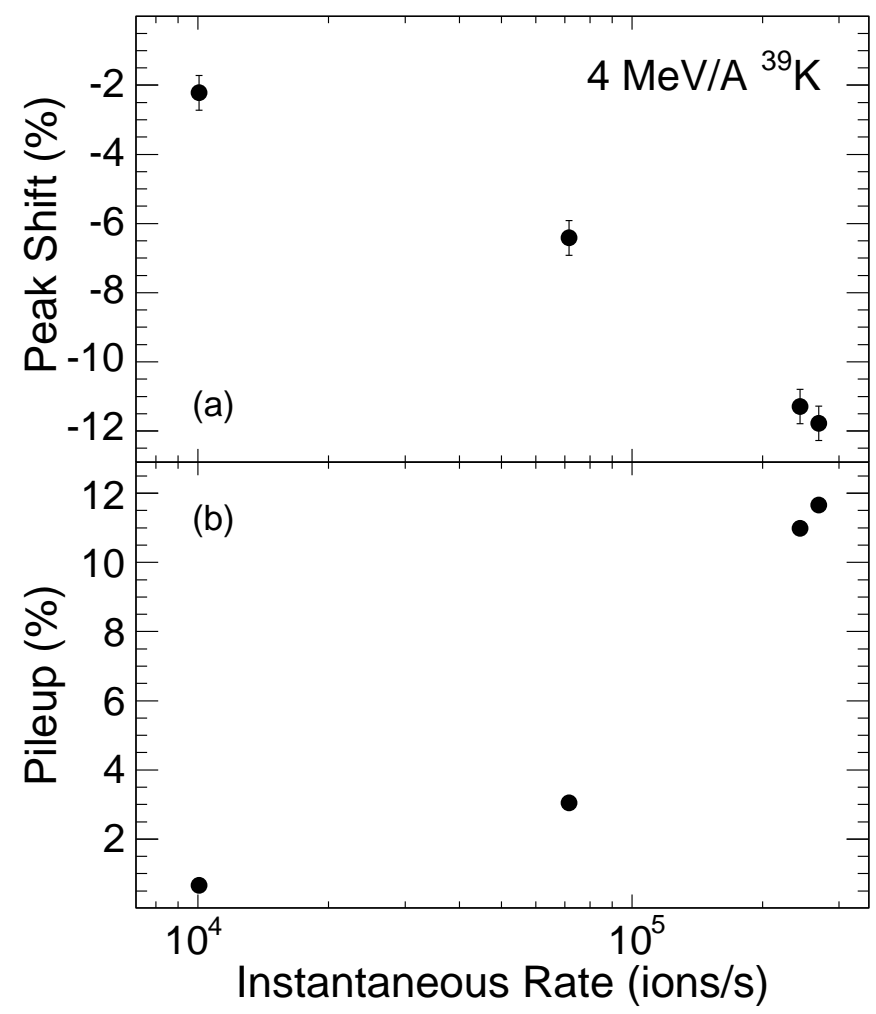

Figure 8: Impact of the beam intensity on the charge collection (panel a) and pileup (panel b) in RIPD. 
Aside from the degradation in the energy resolution the increased beam intensity can also impact the total charge collection due to increased recombination in the detector at high beam intensities. This effect is manifested in the upper panel of Fig. 8. A decrease in the centroid of the energy deposited in RIPD by $4 \mathrm{MeV} / \mathrm{A}{ }^{39} \mathrm{~K}$ ions is evident. With increasing beam intensity the location of the energy centroid shifts to lower values consistent with recombination of electrons and cations in the detector gas. At an instantaneous rate of $3 \times 10^{5}$ ions/s a peak shift of $\sim 12 \%$ is observed as compared to a low intensity beam of 800 ions/s. As recombination impacts both the ions of interest as well as any contaminants, identifying the nuclide of primary interest from other contaminants is still achieveable. However, correcting for any significant changes in the beam intensity becomes important. For the secondary beams that are the principal focus of this detector the beam is often extracted from a charge breeder prior to reacceleration leading to a macro time structure of the beam. Within this extraction pulse the instantaneous beam intensity will typically vary. By recording the extraction time with in the extraction cycle into the data stream this variation in the instantaneous rate can be accounted for.

The impact of beam intensity on the pileup observed is indicated in the lower panel of Fig. 8. Pileup is clearly distinguished as observation of pulses with energies exceeding that of the full beam energy. As expected the pileup increases with increasing instantaneous rate from $<1 \%$ at $1 \times 10^{4}$ ions/s to just under $12 \%$ at $3 \times 10^{5}$ ions/s. As anticipated the fast response of RIPD enables the use of the detector at high rates without significant pileup.

We have also assessed the time resolution of RIPD by measuring the $\Delta \mathrm{E}$ TOF of the beam. The TOF was measured between RIPD and the MCP detector. Due to the sub-nanosecond time resolution of the MCP detector the measured time resolution is dominated by the time resolution of RIPD. The CSA signal was amplified with a timing filer amplifier with integration and differentiation times of 40 and $20 \mathrm{~ns}$ respectively prior to discrimination. The TOF between the MCP and RIPD was measured using a CAEN V1290 TDC 
with a resolution of $25 \mathrm{ps}$. At beam intensities of $<1 \times 10^{3}$ ions/s for an energy deposit of approximately $5.4 \mathrm{MeV}$ a time resolution of $6.8 \mathrm{~ns}$ (FWHM) is obtained. With increasing beam intensity this resolution degrades slightly to $8 \mathrm{~ns}$. For smaller energy deposits in the gas the resolution worsens reaching a time resolution of $13.2 \mathrm{~ns}$ for an energy deposit of $2.3 \mathrm{MeV}$. This time resolution is remarkably good for an ionization chamber and is consistent with the inherently fast risetime and good signal-to-noise evident in Fig.4. This time resolution could be useful in complex multidetector systems as a means to remove pileup events.

\section{Conclusion}

To address the challenge of identifying contaminant species in radioactive isotope beams, we have developed a high-rate axial-field ionization chamber which introduces minimal material into the beam path. The high-rate capability of this detector was optimized by implementing a low profile central anode design and developing a fast charge-sensitive amplifier which is situated within the gas volume in close proximity to the anode. A typical signal from this amplifier spans less than $500 \mathrm{~ns}$, with a rise time of approximately 60-70 ns. The fast response of this detector results in a typical time resolution of 6-8 ns making its timing useful for random/pileup rejection. A fast shaping amplifier was developed to handle these fast signals. With these pulse shaping electronics the energy resolution of RIPD is approximately $8 \%$ for an energy deposit of $3.5 \mathrm{MeV}$ in the detector for $\alpha$ particles from an ${ }^{241} \mathrm{Am}$ source. Using beam, a comparable resolution was obtained. Below an instantaneous rate of $1 \times 10^{5}$

${ }^{39} \mathrm{~K}$ ions/s the energy resolution was $8-10 \%$. At an instantaneous rate of $3 \mathrm{x}$ $10^{5}$ the energy resolution degraded to $14 \%$. The impact of recombination and pileup in the detector as a function of the instantaneous rate was characterized. Correcting for these effects is an important part of exploiting the full capability of this detector to resolve contaminants in a radioactive beam. 


\section{Acknowledgments}

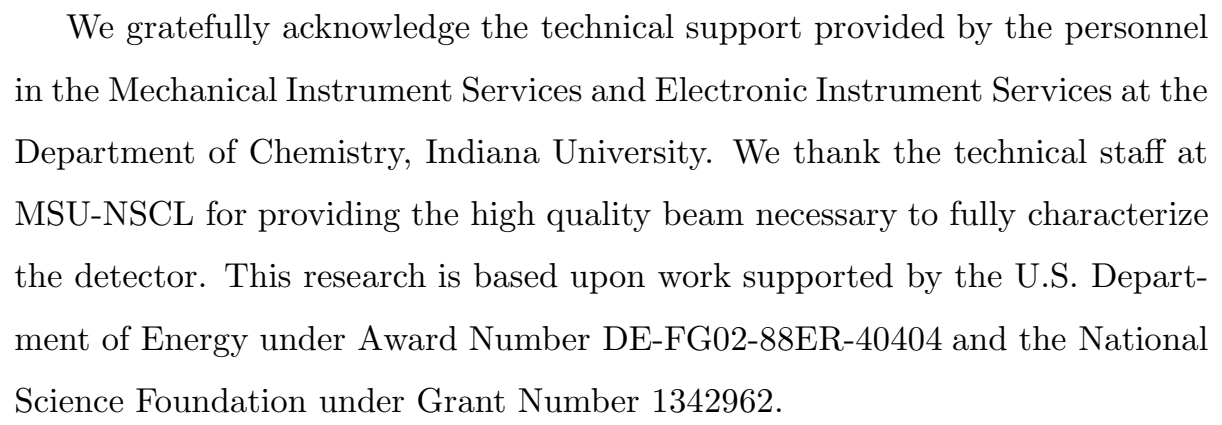

We gratefully acknowledge the technical support provided by the personnel in the Mechanical Instrument Services and Electronic Instrument Services at the Department of Chemistry, Indiana University. We thank the technical staff at MSU-NSCL for providing the high quality beam necessary to fully characterize the detector. This research is based upon work supported by the U.S. Department of Energy under Award Number DE-FG02-88ER-40404 and the National Science Foundation under Grant Number 1342962.

\section{References}

[1] J. F. Liang, et al., Phys. Rev. Lett. 91 (2003) 152701.

[2] A. Lemasson, et al., Phys. Rev. Lett. 103 (2009) 232701.

[3] Y. E. Penionzhkevich, Phys. At. Nucl. 73 (2010) 1460.

[4] T. Glasmacher, Annu. Rev. Nucl. Part. Sci. 48 (1998) 1.

[5] T. J. L. Symons, et al., Phys. Rev. Lett. 42 (1979) 40.

[6] H. L. Ravn, Physics Reports 54 (1979) 201.

[7] Y. T. Oganessian, et al., Nucl. Phys. A 701 (2002) 87.

[8] B. M. Sherrill, et al., Nucl. Instr. Meth. B 56/57 (1991) 1106.

[9] N. Fukuda, et al., Nucl. Instr. Meth. B 317 (2013) 323.

[10] H. W. Fulbright, Nucl. Instr. Meth. 162 (1979) 21.

[11] M. M. Fowler, R. C. Jared, Nucl. Instr. Meth. 124 (1975) 341.

[12] R. W. Zurmuhle, L. Csihas, Nucl. Instr. Meth. 203 (1982) 261.

[13] S. K. Bandyopadhyaya, et al., Nucl. Instr. Meth. A 278 (1989) 467.

[14] K. Kimura, et al., Nucl. Instr. Meth. A 538 (2005) 608. 
${ }_{313}$ [15] K. Y. Chae, et al., Nucl. Instr. Meth. A 751 (2014) 6.

314 [16] T. K. Steinbach, et al., Phys. Rev. C 90 (2014) 041603(R).

315 [17] V. Singh, et al. (2016). arXiv:1603.09314v1 [nucl-ex].

316 [18] J. Va'vra, et al., Nucl. Instr. Meth. A 324 (1993) 113.

317 [19] J. F. Ziegler, M. D. Ziegler, J. P. Biersack, Nucl. Instr. Meth. B 268 (2010)

$318 \quad 1818$

319 [20] T. K. Steinbach, et al., Nucl. Instr. Meth. A 743 (2014) 5. 